\title{
In-Plane Heterostructures Enable Internal Stress Assisted Strain Engineering in 2D Materials
}

\author{
Feng Liu,* Tzu-Chiang Wang, and Qiheng Tang
}

Conventional methods to induce strain in 2D materials can hardly catch up with the sharp increase in requirements to design specific strain forms, such as the pseudomagnetic field proposed in graphene, funnel effect of excitons in $\mathrm{MoS}_{2}$, and also the inverse funnel effect reported in black phosphorus. Therefore, a long-standing challenge in 2D materials strain engineering is to find a feasible scheme that can be used to design given strain forms. In this article, combining the ability of experimentally synthetizing in-plane heterostructures and elegant Eshelby inclusion theory, the possibility of designing strain fields in 2D materials to manipulate physical properties, which is called internal stress assisted strain engineering, is theoretically demonstrated. Particularly, through changing the inclusion's size, the stress or strain gradient can be controlled precisely, which is never achieved. By taking advantage of it, the pseudomagnetic field as well as the funnel effect can be accurately designed, which opens an avenue to practical applications for strain engineering in 2D materials.

\section{Introduction}

In 2004, monolayer graphene was obtained experimentally by mechanical exfoliation ${ }^{[1]}$ giving rise to a surge in searching $2 \mathrm{D}$ materials. Until now, various 2D materials have been found, such as hexagonal boron nitride (h-BN), transition metal dichalcogenides (TMDs), black phosphorus, etc. These novel materials exhibit remarkable electronic, thermal, and optical properties, ${ }^{[2-4]}$ and also show superior mechanical performance, ${ }^{[5]}$ especially their high stretchability, which provides a possibility to manipulate their physical properties via mechanical strain, called strain engineering.

Strain engineering in 2D materials has been intensively studied in the past decade. ${ }^{[6-12]}$ At the moment, there are five conventional ways to induce strain in 2D materials: ${ }^{[12]}$ Bending of a flexible substrate, elongating the substrate, piezoelectric stretching, exploiting the thermal expansion mismatch, and

Dr. F. Liu, Prof. T.-C. Wang, Prof. Q. Tang

State Key Laboratory of Nonlinear Mechanics

Institute of Mechanics

Chinese Academy of Sciences

Beijing 100190, China

E-mail: liufeng@imech.ac.cn

Prof. T.-C. Wang, Prof. Q. Tang

School of Engineering Science

University of Chinese Academy of Sciences

Beijing 100049, China

DOI: 10.1002/smll.201703512 controlled wrinkling. Despite a variety of methods to induce strain, the strain forms realized in 2D materials until now are constricted to three modes, i.e., uniaxial and biaxial uniform tensile strain as well as the nonuniform strain induced by wrinkling, which could hardly satisfy the sharply increased requirements to design specific strain forms, such as the pseudomagnetic field proposed in graphene, ${ }^{[10]}$ funnel effect of excitons in $\mathrm{MoS}_{2},{ }^{[13]}$ and also inverse funnel effect reported in black phosphorus several months ago. ${ }^{[14]}$ In this sense, the progress in experiments lags far behind theoretical predictions restricting the developments of strain engineering in 2D materials, and thus a long-standing challenge in this field is to find a feasible scheme that can be used to design the given strain forms in $2 \mathrm{D}$ materials.

Relative to the dilemma faced in strain engineering, the synthesis experimental technique for 2D materials has made rapid progress recently. Especially, the in-plane heterostructures could be designed to aimed geometries assisting the study of their novel properties. Typical examples are the in-plane graphene/ h-BN heterostructure ${ }^{[15-22]}$ and TMDs heterostructures such as

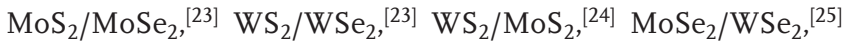
and $\mathrm{WSe}_{2} / \mathrm{MoS}_{2} \cdot{ }^{\left[{ }^{26]}\right.}$ As the pattern design for in-plane $2 \mathrm{D}$ heterostructures becomes now available, the internal stress and strain states in the system could be controlled in terms of meso-mechanics theory, which provides an opportunity to overcome the long-standing challenge for strain engineering in $2 \mathrm{D}$ materials.

In this article, we demonstrate that stress or strain states in 2D materials could be accurately designed by utilizing the elegant Eshelby inclusion theory. ${ }^{[27]}$ Although Eshelby inclusion theory has been discussed long ago in mesomechanics, it is usually used to analyze stress and strain states of bulk materials embedded with inclusions. As inclusions are exposed outside in 2D materials, a new feature introduced in Eshelby inclusion theory is to design given strain $\backslash$ stress fields. Indeed, it is found that with the designed patterns of heterostructures, internal stress or strain states could be induced and accurately controlled at nanoscale to manipulate physical properties. Comparing with general strain engineering, here not only external forces but also the internal stress could be utilized to design strain states for tuning physical properties, and thus it is called internal stress assisted strain engineering. With internal stress assisted strain engineering, arbitrary uniform tensile strain 
forms could be obtained. Even more amazing, as there is no characteristic length scale in Eshelby inclusion theory, the stress and strain distribution only depends on the shape of the inclusion, implying that the stress or strain gradient could be controlled precisely through changing the inclusion's size, leading to an accurate design for pseudomagneitc field and funnel effect. In the following, graphene/graphane heterostructures will be taken as an example to show the power and versatility of our internal stress assisted strain engineering.

\section{Resutls and Disscusion}

\subsection{Accurate Designs for Uniform Strain Forms}

Consider an infinite linear elastic body contains an inclusion region, and in this region some physical or chemical transformations (such as localized thermal expansion and phase transition) take place, which induce an initial transformation strain. To find out the elastic state due to the initial transformation strain inside the inclusion is called the transformation problem. As the initial transformation strain is often called eigenstrain, the transformation problem could also be called eigenstrain problem. Through a thought experiment, Eshelby obtained the solution to the eigenstrain problem. Note that in this problem, the elastic constants are the same for the inclusion and matrix. However, in a normal inclusion's problem, the elastic constants of the inclusion are different from those of the matrix. Fortunately, equivalence could be established between these two problems (i.e., the eigenstrain problem and normal inclusion's problem), so that the solution given by Eshelby could also be used to obtain the stress and strain distributions in the normal inclusion's problem, which is called Eshelby inclusion theory. A wellknown result from Eshelby inclusion theory is that the stress and strain field inside an ellipsoidal inclusion is uniform the proof is reproduced in the Supporting Information). This conclusion remains in two dimensions, i.e., for 2D materials, a uniform strain state could be obtained in an elliptic inclusion area (the proof could be found in the Supporting Information). Thanks to the electron-stimulated desorption technique, the adsorbed hydrogen atoms could be desorbed selectively in experiments leading to given patterns with nanometer scale resolution for graphene/graphane in-plane heterostructures. ${ }^{[28]}$ Therefore, with an elliptic heterostructure design the uniform strain states are actually now experimental achievable in this system according to Eshelby inclusion theory. In this section, we will show how to accurately control the uniform strain form via the pattern design of the graphene/graphane heterostructure. Note that due to the large numbers of atoms involved, first principle calculations are nearly impossible to handle the inclusion problem, however, with mature empirical potentials, molecular dynamics (MD) simulations become a good choice and will be used in our study.

In Figure 1a, the atomic structures for graphene (upper) and graphane (lower) are plotted. Among all possible configurations for graphane, the most stable one (chair configuration) ${ }^{[29]}$ (a)

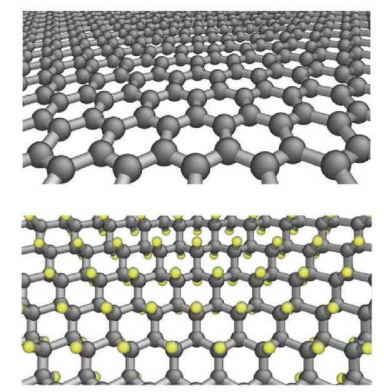

(d)

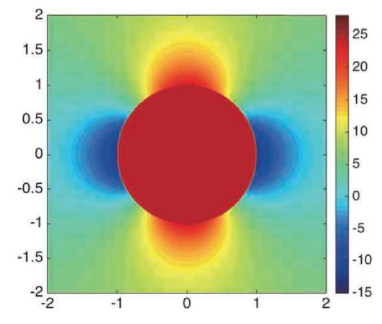

(b)

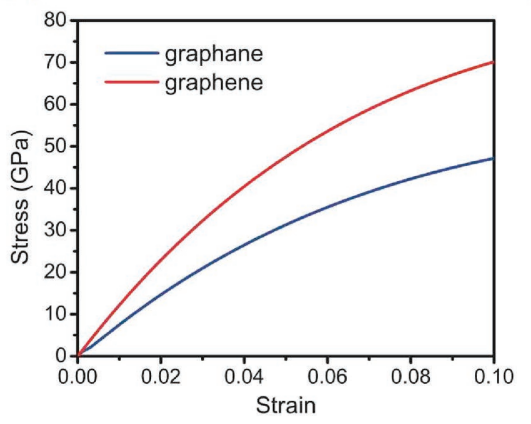

(e)

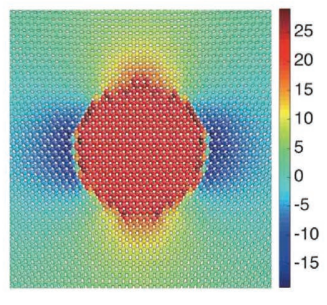

(c)

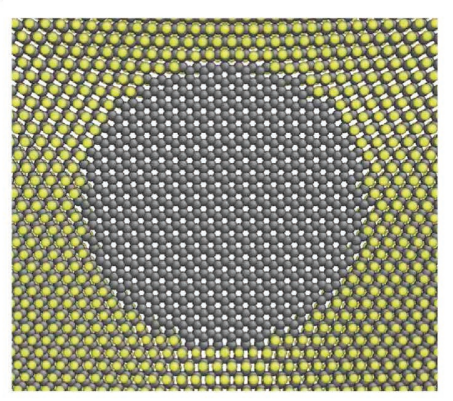

(g)

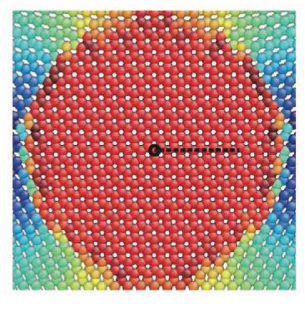

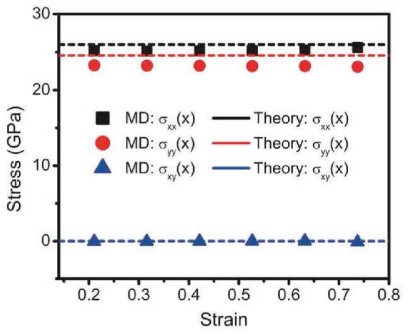

Figure 1. The stress distribution for the circular graphene/graphane heterostructure. a) The atomic structure of graphene (upper) and graphane (lower), where the gray and yellow colored atoms represent carbon and hydrogen atoms, respectively. b) The uniaxial tensile tests are preformed in MD simulations, and the obtained stress-strain curves for graphene and graphane are shown. c) The top view of the circular graphene/graphane heterostructure model established in our MD simulations. d) The distribution of the stress component $\sigma_{y y}$ obtained from inclusion theory of Eshelby, and the color reflects its magnitude and sign. e) The distribution of the stress component $\sigma_{y y}$ obtained from MD simulations, and in panel ( $\mathrm{f}$ ) the inclusion region is zoomed in. g) Along with the dashed line marked in panel (f), the stress components obtained from two methods, i.e., inclusion theory of Eshelby and MD simulations, are compared. 
is applied here, which is obtained by decorating graphene with hydrogen atoms on both sides alternately. After sufficient relaxations, tensile tests are performed in MD simulations for graphene and graphane, in terms of which the stress-strain curves are obtained (Figure 1b) and the mechanical quantities used in Eshebly inclusion theory could be quantified (details could be found in the Experimental Section). To confirm the validity of Eshebly inclusion theory, a circular graphene/graphane heterostructure model is established by removing the hydrogen atoms inside a circular region from graphane, and its top view is shown in Figure 1c. The distribution of stress component $\sigma_{Y Y}$ around the circular inclusion according to Eshebly inclusion theory is plotted in Figure 1d (the inclusion problem is solved numerically, and the details can be found in the Experimental Section), and for comparison the atomic stress $\sigma_{Y Y}$ distribution obtained from MD simulations is given in Figure 1e (the zooming circular inclusion region is shown in Figure 1f). There are two things, which should be noticed: first, hydrogen atoms are invisible in Figure 1e,f to clarify the stress distribution of the carbon frame, which undertakes almost all of loads, and the following stress distributions will be plotted in this way unless otherwise noted; second, the sample in MD simulations is subject to $1 \%$ external biaxial tensile strain, and the corresponding external stress is used to set the stress boundary conditions in the inclusion problem. It is obvious that the stress distribution from MD simulations agrees well with theoretical results, especially the uniform stress distribution in the circular inclusion region. To see it quantitatively, the stress distribution in the circular inclusion region along the $x$-direction is shown in Figure $1 \mathrm{~g}$, where the little derivation between $\sigma_{x x}$ and $\sigma_{y y}$ stems from the slight mechanical anisotropy along different lattice orientations. It is surprising to find that the theoretical prediction could give a pretty good description of the stress distribution. More importantly, the theoretically predicted uniform stress/strain state could be well reproduced in a nanoscale system, which gives us much confidence that an accurate design of strain forms in nanodevices could be possible in experiments.

The biaxial uniform strain state realized in the circular heterostructure model is actually not new, since it could be obtained by conventional methods, such as piezoelectric stretching or exploiting the thermal expansion mismatch. Here the challenge is to design new uniform strain forms, which are significant to experimentally clarify connections between physical properties and mechanical strain. As stated in Eshelby inclusion theory, the strain distribution is uniform inside an elliptic inclusion, based on which we could design new uniform strain forms by controlling the shape and also the orientation of the elliptic inclusion.

The elliptic graphene/graphane heterostructure model is shown in Figure 2a. Comparing with the circular one, there are additional freedoms in this model, i.e., the shape and the orientation of the ellipse. Here $m=(a-b) /(a+b)$ is used to define (a)

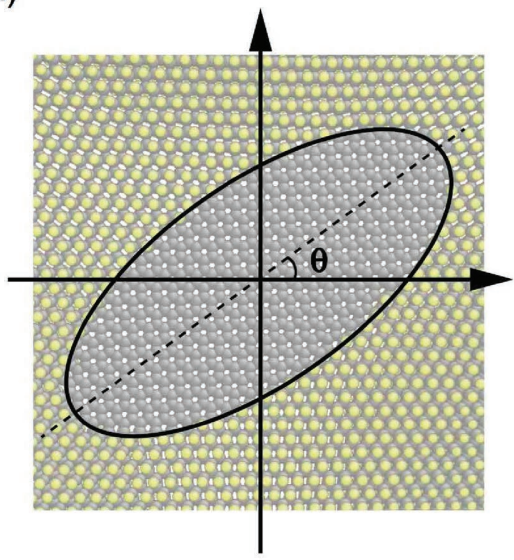

(c)

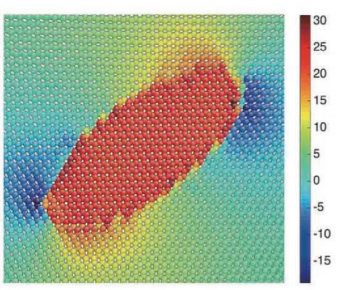

(b)

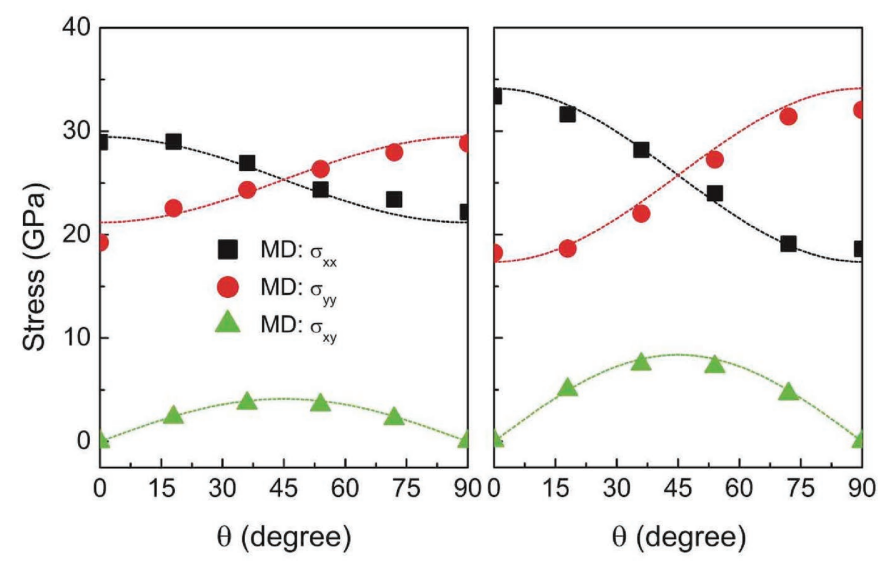

(d)

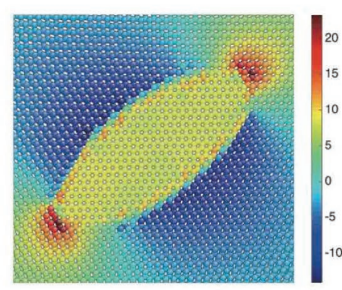

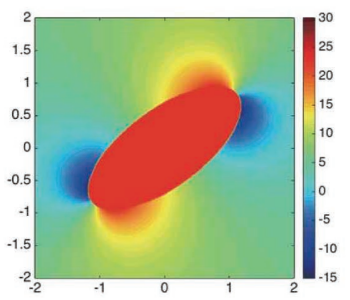

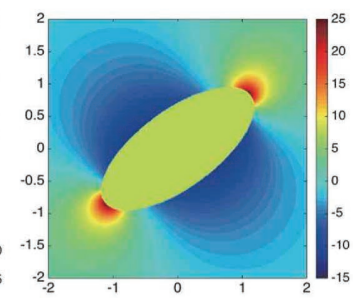

Figure 2. The stress distribution for the elliptic graphene/graphane heterostructure. a) The top view of the elliptic graphene/graphane heterostructure model established in our MD simulations, where the gray and yellow colored atoms represent carbon and hydrogen atoms, respectively. Note that $\theta$ is the angle between the long axis of ellipse and the $x$-axis. b) The variation of stress components with respect to $\theta$ with $m$ equals 0.2 (left) and 0.4 (right), where the dots are obtained from MD simulations and the dashed lines are calculated according to the Eshelby inclusion theory. c) The distributions of stress components $\sigma_{y y}$ (left) and $\sigma_{x y}$ (right) obtained from MD simulations, where $\theta=36^{\circ}$ and $m=0.4$. d) The distributions of stress components $\sigma_{y y}(\mathrm{left})$ and $\sigma_{x y}$ (left) obtained from inclusion theory of Eshelby, where $\theta=36^{\circ}$ and $m=0.4$. 
the shape of the ellipse, where $a$ and $b$ represent the length of the long and short axis, and $\theta$ describes the orientation of the ellipse, which is marked in Figure 2a. The samples subject to $1 \%$ biaxial tensile strain are simulated via MD simulations, and the stress components $\left(\sigma_{x x}, \sigma_{y Y}\right.$, and $\left.\sigma_{x y}\right)$ inside the elliptic inclusion as functions of the angle $\theta$ are shown in Figure $2 \mathrm{~b}$ with $m$ equaling 0.2 (left) and 0.4 (right), respectively. It is important to note that in MD simulations, the values of these stress components are taken from the central atom, since the stress distribution is uniform inside the inclusion no matter for Eshelby inclusion theory or MD simulations. With $1 \%$ biaxial strain boundary condition set in the inclusion problem, the theoretical prediction (the dashed lines in Figure $2 \mathrm{~b}$ ) agrees quite well with the MD simulations (the dots in Figure 2b).

As our goal is to obtain new uniform stress $\backslash$ strain states in 2D materials, it should first be able to distinguish stress $\backslash$ strain states. Here, the values of principal stresses are used to do this job. It is found that with fixed applied biaxial tensile strain, $\theta$ and $m$ determine the direction and values of principal stresses, respectively. To be specific, in Figure $2 b$, the values of principal stresses remain for different $\theta$, which can be found by solving the eigenvalues of the stress tensor, while with $m$ increasing, the difference between two principal stresses components becomes bigger, and the corresponding stress $\backslash$ strain form could change from biaxial tensile stretching $(m=0)$ to uniaxial tensile stretching $(m=1)$. It is surprising to find out that in principle, arbitrary uniform tensile strain forms could be designed by tuning $m$, i.e., the shape of the elliptic inclusion. With $\theta=36^{\circ}$ and $m=0.4$ for the elliptic heterostructure, the distributions of stress components $\sigma_{Y y}$ and $\sigma_{x y}$ obtained from MD simulations (Figure 2c) and theoretical predictions (Figure 2d) are shown, which once again demonstrates that the stress/strain states could be well predicted in terms of Eshelby inclusion theory.

In fact, the method proposed here is compatible with previous methods (e.g., elongating the substrate, piezoelectric stretching, etc.), with the assistance of which not only the form but also the magnitude of the uniform strain state could be accurately designed. It is therefore called internal stress assisted strain engineering that could provide a new avenue for this field.

\subsection{Nonuniform Strain-Pseudomagnetic Field}

The issue about opening an energy gap in graphene has always been discussed since its birth. Until now, various methods have been proposed including periodically structural modification, ${ }^{[30-34]}$ quantum confinement, ${ }^{[35]}$ chemical modification, ${ }^{[36,37]}$ etc. However, all of these methods lack controllability, since the energy gap could be hardly manipulated after these modifications. This situation does not change until the concept of pseudomagnetic field is introduced by Guinea et al. ${ }^{[10]}$ In this paper, they argue that a nonuniform strain field could induce an effective magnetic field, called pseudomagnetic field, by making use of which the energy gap in graphene could be opened. To generate a spatial uniform energy gap in graphene, a spatial uniform pseudomagnetic field is required, which means stress field should satisfy the following relation ${ }^{[10]}$

$\sigma_{x x}=-\sigma_{y y}=C y, \quad \sigma_{x y}=C x$ where $C$ is a constant. Unfortunately, this specific nonuniform strain forms could not be induced by conventional ways. Despite in experiments, over $300 \mathrm{~T}$ pseudomagnetic field (nearly uniform) has been observed on graphene nanobubbles, ${ }^{[38]}$ the uncontrollability makes it impossible to be adopted in industrial design. In the following, we will show that with internal stress assisted strain engineering a nearly uniform pseudomagnetic field could be realized.

In Figure 3, the triangular graphene/graphane heterostructure is designed to realize uniform pseudomagnetic field in graphene. With a theoretical calculation, the stress $\sigma_{\gamma Y}$ distribution (1\% biaxial tensile strain is assumed at infinity) is shown in Figure 3a. Since there is no characteristic length scale in Eshelby inclusion theory, in principle triangular inclusion regions with different sizes should give the same distribution if the distance is normalized with the length scale of triangle. To confirm this issue, MD simulations are performed. Two triangular heterostructure models with their inclusion regions being different in size are established, and the distribution of stress component $\sigma_{Y Y}$ for the larger triangular inclusion and the smaller one can be found in Figure 3b,c, respectively. To quantitatively compare MD simulations with Eshelby inclusion theory, all of the stress components distribution along the $x$ - and $y$-directions (the route is marked in Figure 3d) in the inclusion region obtained by the two methods are given in Figure 3e,f. Note that although both two models in MD simulations are subject to $1 \%$ external biaxial tensile strain, the corresponding external stresses are slightly different, which are considered and used to set the stress boundary conditions in the inclusion problem. In general, MD simulations agree well with Eshelby inclusion theory for both cases, and especially for the model with smaller inclusion region, where two methods nearly completely match with each other (Figure $3 \mathrm{f}$ ). There are two things should be noticed. First, Eshelby inclusion theory describes the smaller triangular inclusion better than the larger one, since with the same size of the simulation box, the boundary effect has less influence to the smaller triangular inclusion area relatively, in other word, it is more close to the infinite body assumption in Eshelby inclusion theory. Second, the size effect is nearly negligible, since both the larger and smaller triangular inclusion regions could be well described by the Eshelby inclusion theory, in which there is no characteristic length scale. By following the procedures introduced in the Experimental Section, the atomic stress in MD simulations is used to calculate the pseudomagetic field for the smaller triangular inclusion sample, which is nearly uniform and up to $140 \mathrm{~T}$ as shown in Figure $3 \mathrm{~g}$. Note that the local value of pseudomagnetic field is obtained by interpolation and then taking its average within an area of $1.6 \AA$ (slight longer than the $\mathrm{C}-\mathrm{C}$ bond length) around, so that the discreteness brought by the atomic structure could be eliminated. The pseudomagetic field according to Eshelby inclusion theory is shown in Figure $3 \mathrm{~h}$ for comparison. As can be seen, the results from MD simulations and Eshelby inclusion theory are consistent, and the major difference occurs at the sharp atomic interface, which could be accepted by noting that the Eshelby inclusion theory is based on the continuum assumption (more comparisons in details could be found in the Supporting Information). Since there is no characteristic length scale in Eshelby inclusion theory, with the inclusion size 
(a)

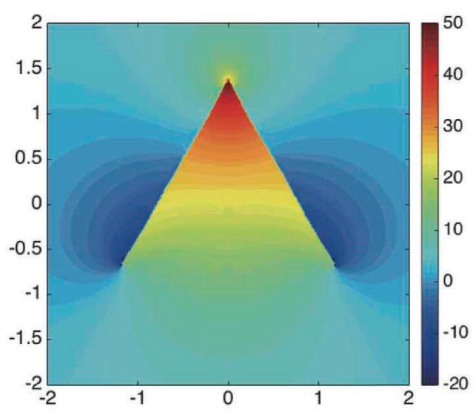

(b)

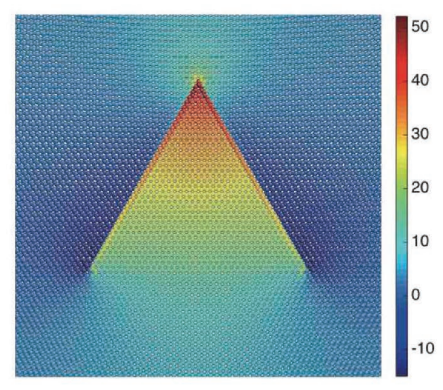

(c)

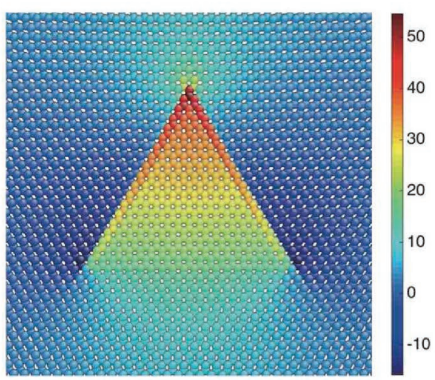

(d)

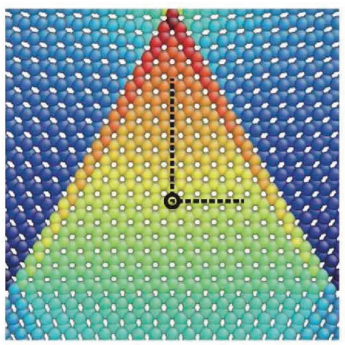

(g)

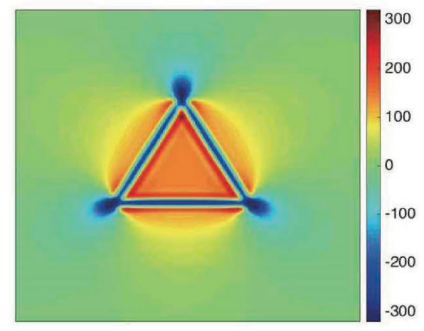

(e)

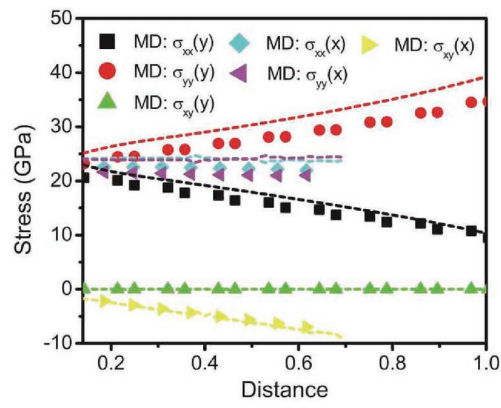

(h)

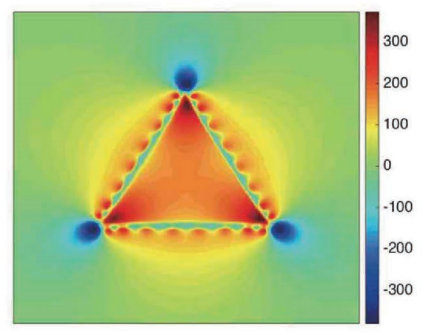

(f)

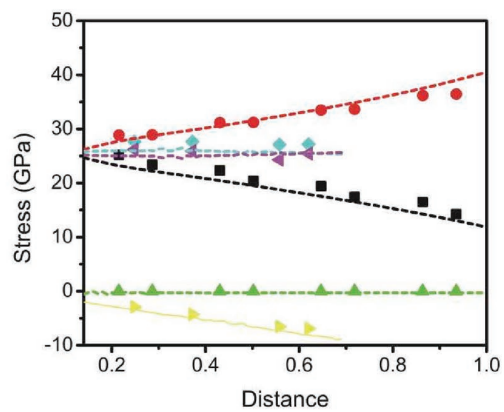

(i)

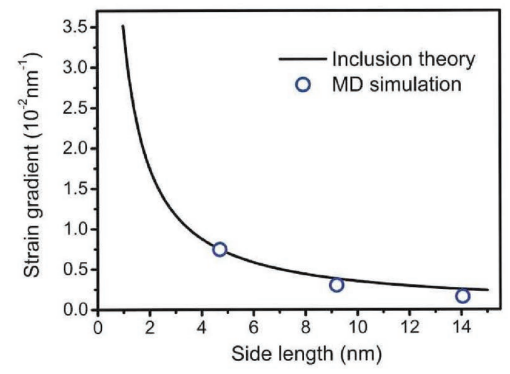

Figure 3. Pseudomagnetic field for the triangular graphene/graphane heterostructure. a) The distribution of stress components $\sigma_{y y}$ obtained from the inclusion theory of Eshelby. The distributions of stress components $\sigma_{y y}$ for b) the larger triangular inclusion sample and c) the smaller one obtained from MD simulations. Note that the side length of the triangle is about 9.2 and $4.7 \mathrm{~nm}$ for the larger and smaller one, respectively. d) The inclusion region of the smaller triangular inclusion model is zoomed in. Along the $x$ - and $y$-direction (the route is marked with black dashed lines in panel (d)), stress components obtained from two methods, i.e., inclusion theory of Eshelby and MD simulations, are compared for e) the larger triangular inclusion model and $f$ ) the smaller one, respectively. Note that the distance is normalized with the size of the triangle. The corresponding pseudomagnetic fields for the smaller triangular inclusion model obtained by g) MD simulations and $h$ ) Eshelby inclusion theory are shown. i) The variation of the strain gradient (the average value of $\partial \sigma_{y \gamma} / \partial y$ inside the triangle) with respect to the side length of the triangular inclusion.

normalized, the stress $\backslash$ strain distribution remains unchanged. As a result, the magnitude of the stress $\backslash$ strain gradient should be inversely proportional to inclusion size. Therefore, in experiments, triangular heterostructure patterns with different sizes could be used to obtain the uniform and controllable strain gradient. To confirm this point of view, the variation of strain gradient (the average value of $\partial \sigma_{Y Y} / \partial y$ inside the triangle) with respect to the side length of the triangular inclusion is shown in Figure 3i, and there is a good agreement between MD simulations and Eshelby inclusion theory. It demonstrates that with the triangular heterostructure design proposed in this article, the strain gradient and thus pseudomagnetic field could be accurately designed.
One thing should be noted is that although the fundamental equations (i.e., equilibrium equations, constitutive equations, etc.) are the same, the boundaries conditions in this article are quite different from ref. [10], which makes that our results presented in Figure $3 \mathrm{~g}$ are distinct from those reported in ref. [10]. Here the force boundary conditions are applied on graphene, which is direct but hard to realize in experiments. This is because only uniaxial and biaxial stretching are now available in experiments, which cannot afford the accurate force boundary conditions setting in ref. [10]. However, combing the triangular in-plane heterostructure design with the biaxial external stretching, internal stress assisted strain engineering enables to realize a nearly uniform pseudomagnetic field. 


\subsection{Nonuniform Strain-Funnel Effect}

The funnel effect of excitons in $\mathrm{MoS}_{2}$ monolayers, a potential application for strain engineering in photonics, has been reported by Feng et al. ${ }^{[13]}$ recently. They demonstrated that strain gradients could induce the continuous varying profile of optical gap in $\mathrm{MoS}_{2}$, which serves as a force that could push excitons toward the regions of maximum tension. This concept could enhance the efficiency for solar energy harvesting and lead to significant performance gains in photovoltaic solar cells. Without much time, the existence of the funnel effect in $\mathrm{MoS}_{2}$ has been verified in experiments, ${ }^{[39,40]}$ and several months ago, inverse funnel effect of excitons has been theoretically predicted in black phosphorus, ${ }^{[14]}$ which suggests this effect could be ubiquitous in 2D materials. Although much attention has been paid in theory and experiment, now only two methods, i.e., winkling ${ }^{[39]}$ and indentation, ${ }^{[40]}$ enable to experimentally realize the funnel effect. Since both of them take advantage of the interaction between the substrate and 2D materials, the properties of 2D materials could be affected. Here we will take the graphene/graphane heterostructure as an example to show that with the internal stress assisted strain engineering, the funnel effect in 2D materials could be realized without the assistance of the substrate, and thus should be convenient for applications.

To this end, the strain dependence of the gap should be clarified first. Take monolayer $\mathrm{MoS}_{2}$ as an example, in this system $1 \%$ strain could lead to $45 \mathrm{meV}$ variation for energy gap according to the pervious experimental studies, ${ }^{[26,39,41-45]}$ which means the tensile and compressive strain give an equivalent variation of the gap except the opposite signs. Note that since only some simple strain forms could be realized in experiments, the strain dependence of the gap cannot be completely determined. If we assume that the trace of strain tensor is proportional to the variation of the energy gap, except at the interface between the inclusion and matrix, the gradient for the trace of strain tensor and thus the variation of the bandgap will vanish according to Eshelby inclusion theory (the proof could be found in the Supporting Information). To overcome this problem, the compressive instability of $2 \mathrm{D}$ materials, which leads to asymmetric mechanical responses for tension and compression, should be made use of. Note that here we focus on realization of a nonzero gradient for the trace of strain tensor, and as mentioned above, the graphene/graphane heterostructure will be taken as a model system to show how this could be accomplished.

In Figure 4a, a circular graphene/graphane heterostructure model for MD simulations is established, and the distribution for the trace of stress $\backslash$ strain (subject to $0.5 \%$ external biaxial tensile strain) is shown in Figure $4 \mathrm{~b}$. Due to the compressive instability, the inclusion region is protruding (see the Figure $4 \mathrm{a}$ left), and the nonzero gradient for the trace of strain tensor could be observed in Figure 4b. Note that the graphene area outside the inclusion region is focused, and the corresponding spatial variation of the trace of strain tensor along the $x$-direction is shown in Figure 4c, which is remarkable when the distance is comparable with the radius of the circular inclusion region. The typical band structure of $\mathrm{MoS}_{2}$ according to density functional theory (DFT) calculations with Perdew-Burke-Ernzerhof exchange-correlation potential ${ }^{[46]}$ is shown in Figure $4 d$, and its unit cell and first Brillouin zone are given in the insets of Figure $4 \mathrm{~d}$. Here, the rectangle unit cell is chosen to facilitate applying strain. For the typical band structure of $\mathrm{MoS}_{2}$, three relevant energies are marked with $\mathrm{V}, \mathrm{C}$, and $\mathrm{H}$ in Figure $4 \mathrm{~d}$, respectively. To demonstrate that the designed strain field could induce the funnel effect in $\mathrm{MoS}_{2}$, the evolution of the bandgap in $\mathrm{MoS}_{2}$ is studied via DFT calculations with the exchange correlation potential chosen the hybrid functional HSE06 [47,48] (calculation details could be found in the Experimental Section). Note that the strain state is extracted from the circular graphene/graphane heterostructure, and then used to calculate the band structure of $\mathrm{MoS}_{2}$. The energy variation of $V, C$, and $H$, and the evolution of the direct gap (the energy difference between $C$ and $H$ ) and indirect gap (the energy difference between $C$ and $V$ ) are shown in Figure 4e,f, respectively. It could be seen that the energy of conduction band minimum (CBM), the highest occupied energy at L, and the direct gap increase with distance, which shows an inverse trend with the trace of stress and strain. Combining with the high and strain insensitive exciton binding energy (about $0.5 \mathrm{eV}$ ) reported in $\mathrm{MoS}_{2}{ }^{[13]}$ Type III scenario proposed in ref. [13] is satisfied. Therefore, the designed stress $\backslash$ strain field could be used to realize the funnel effect in $\mathrm{MoS}_{2}$. Since the assistance from substrates now becomes unnecessary and only simple external loads (i.e., biaxial stretching) need to be applied, this method should be friendly to various applications.

The only question left is how to design the pattern of heterostructure in experiments for these systems. With the successful functionalization of $\mathrm{MoS}_{2}{ }^{[49]}$ and black phosphorus ${ }^{[50]}$ reported recently as well as the simple triangular and hexagonal TMDs heterostructures with nanometer resolution obtained in experiments, ${ }^{[23-26]}$ two possible ways could be chemical modification and epitaxial growth for heterostructures. Considering the rapid development of experimental technology, accurately patterning in these systems should be expected in near future.

\subsection{Spatial Uniform Distributed Bandgap in the Triangular $\mathrm{MoS}_{2} / \mathrm{WSe}_{2}$ Heterostructure}

In Figure 5a, the schematic diagram for the $\mathrm{MoS}_{2} / \mathrm{WSe}_{2}$ in-plane heterostructure in experiments ${ }^{[26]}$ is shown, where an interesting observation is that the spatial distribution of bandgap is uniform in the $\mathrm{WSe}_{2}$ region. Here, we try to explain this phenomenon via theoretical analysis. Note that in theoretical analysis, the solution of infinite body is used, since the finite boundary effect plays little role in the stress distribution inside the inclusion, which will be demonstrated in the following.

With the lattice constant of $\mathrm{WSe}_{2}(0.328 \mathrm{~nm})^{[51]} 2.5 \%$ larger than $\mathrm{MoS}_{2}(0.32 \mathrm{~nm}),{ }^{[52]}$ the eigenstrain equals $2.5 \%$ suggesting that the inclusion is subject to compression. As their elastic properties have been studied ${ }^{[53-55]}$ (Young's modulus and Poisson's ratio are $270 \mathrm{GPa}$ and 0.24 for $\mathrm{MoS}_{2}, 251.64 \mathrm{GPa}$ and 0.19 for $\mathrm{WSe}_{2}$ ), the stress or strain state of the heterostructure could be obtained according to Eshelby inclusion theory. The distribution of stress components $\sigma_{x x}$ and $\sigma_{x y}$ is plotted in Figure 5b,c, and their spatial variation along the middle line (marked with 
(a)

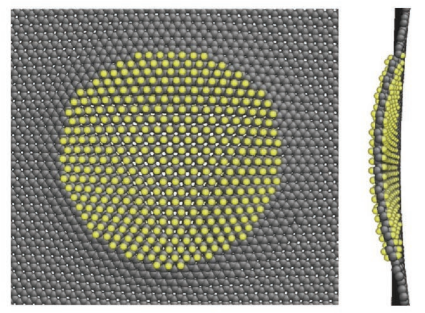

(b)

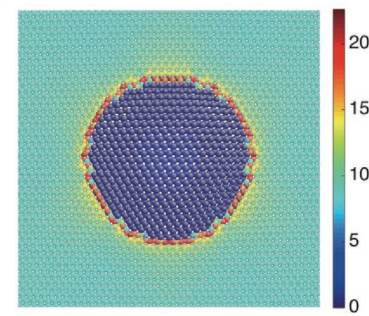

(d)

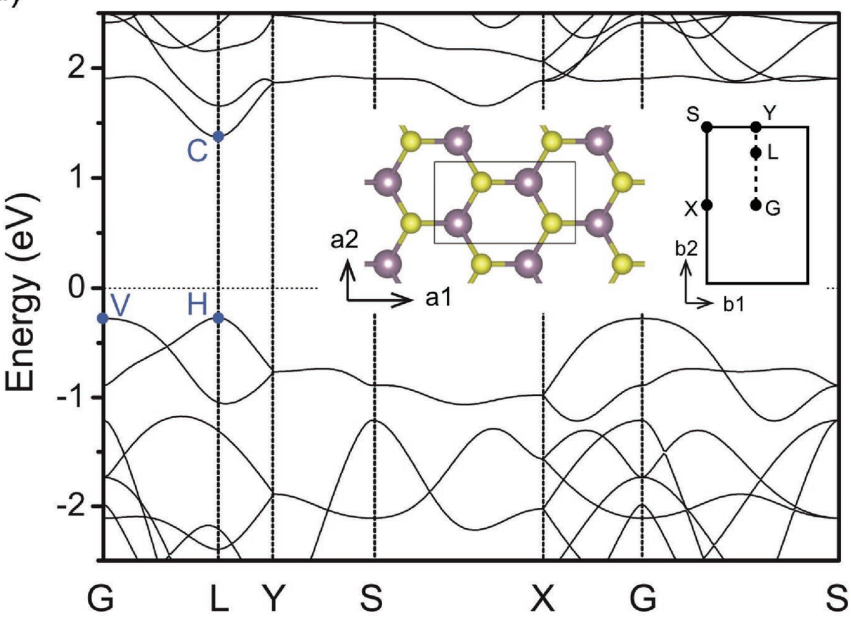

(c)

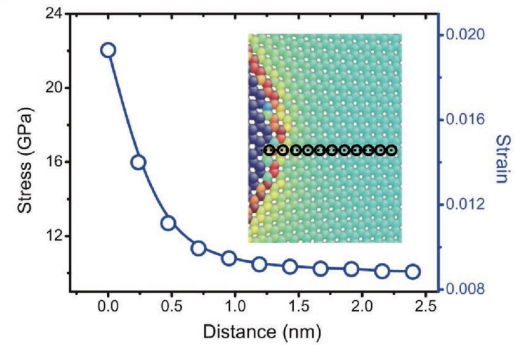

(e)

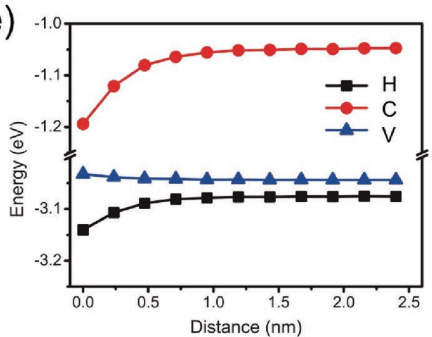

(f)

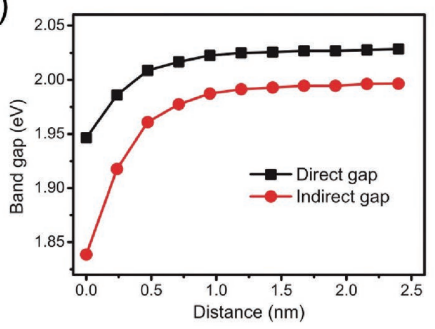

Figure 4. The funnel effect realization. a) The top view (left) and side view (right) of the circular graphene/graphane heterostructure model, where the gray and yellow colored atoms represent carbon and hydrogen atoms, respectively. Note that in the side view, the cross-section of this model is shown. b) The distribution for the trace of stress tensor obtained from MD simulations, and it is set to zero in inclusion region, which is not interested in this case. c) The variation of the trace of the stress tensor along the $x$-direction with respect to the distance from the interface of the heterostructure. Note that the atoms in the path are marked with black circles in the inset. d) The typical band structure of monolayer MoS $\mathrm{S}_{2}$ without strain is shown, and three relevant energies are marked with $V, C$, and $H$, respectively. Insets: the unit cell and first Brillouin zone. e) The dependence of $V, C$, and $H$ with respect to the distance from the interface of the heterostructure. $f$ ) The corresponding evolution of the direct gap (the energy difference between $C$ and $H$ ) and indirect gap (the energy difference between $C$ and $V$ ) are shown.

the red dashed line in Figure 5b,c) is plotted in Figure 5d, the maximum magnitude of which is about $8 \mathrm{GPa}$. In Figure 5e, the normal and tangential stress along one side of the triangle (marked with the black dashed line in Figure 5b,c) are given, as their magnitude are much smaller than that of the stress in the inclusion area, finite boundaries should have little effect for the stress distribution in the inclusion region. The trace of stress tensors has been plotted in Figure 5f, which is uniform in terms of Eshelby inclusion theory. Different from Figure 4b, here the compressive instability could be avoided due to the assistance from the substrate. Providing that the trace of strain tensor is proportional to the variation of the energy gap for $\mathrm{WSe}_{2}$, the spatial distribution of the bandgap should be uniform, which is just the case observed in experiments. ${ }^{[26]}$

One relevant issue is whether the lattice mismatch near the interfaces of the heterostructures could induce dislocations to release the strain. Due to strong covalent bonds, dislocations could hardly slip in 2D materials, and for this reason most of 2D materials lack plasticity at room temperature. Despite there is a lattice mismatch near the interfaces of the in-plane heterostructures, at low temperature it will not induce dislocations to release the strain. This is because the motions of dislocations are nearly forbidden in this situation, and as a result the internal strain field should be well reserved.

Thanks to the rapid progress of experimental technique in recent years, in-plane heterostructures could be synthetized to aimed geometries. Recently, it has been reported that simple hexagonal and triangular patterns could be obtained for in-plane 2D TMDs heterostructures. ${ }^{[23-26]}$ Not only that, but the pattern could be designed at nanoscale to write letters in graphene/graphane heterostructure ${ }^{[28]}$ and even draw an owl in graphene/BN heterostructure. ${ }^{[15]}$ With all these experimental advances, the broad applying prospect for internal stress assisted strain engineering might be right around corner.

\section{Conclusion}

In conclusion, with the Eshelby inclusion theory, the internal stress/strain could be utilized to accurately design strain states. From a fundamental perspective, with tuning the pattern of heterostructures, internal stress assisted strain engineering could be used to accurately design any uniform tensile strain field or precisely control the strain gradient in the nonuniform 
(a)

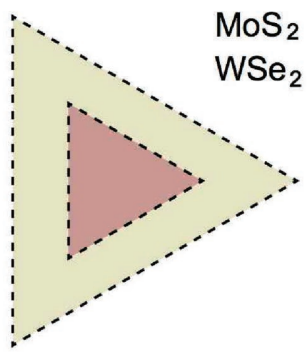

(d)

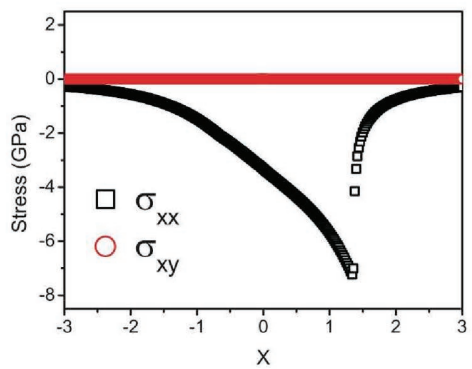

(b)

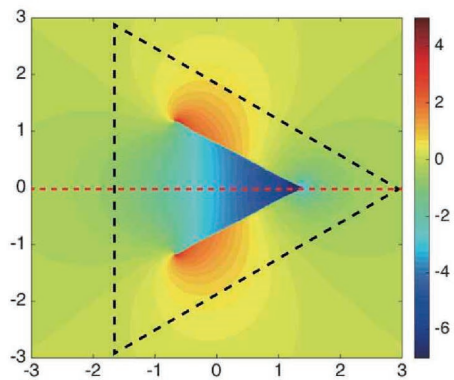

(e)

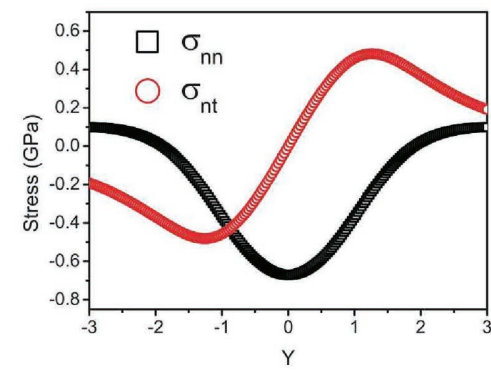

(c)

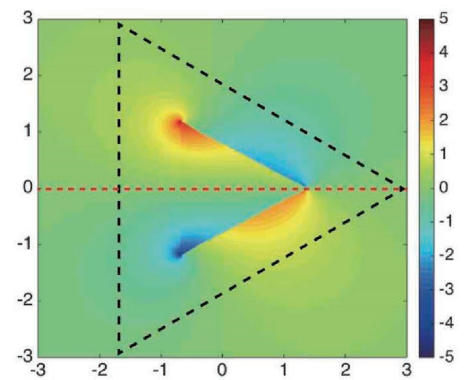

(f)

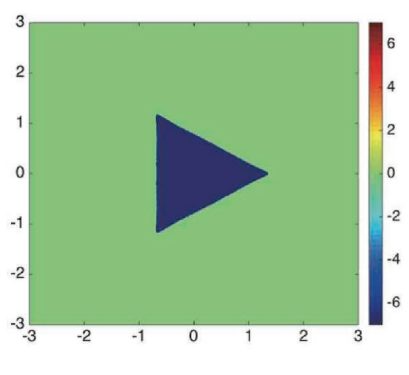

Figure 5. The uniform distribution of the trace of the stress tensor in triangular $\mathrm{MoS}_{2} / \mathrm{WSe}_{2}$ heterostructure obtained in experiments. a) The triangular $\mathrm{MoS}_{2} / \mathrm{WSe}_{2}$ heterostructure obtained in experiments. ${ }^{[26]}$ The distribution of the stress components $\sigma_{x x}$ and $\sigma_{x y}$ obtained from the inclusion theory of Eshelby is shown in panels (b) and (c), respectively. d) The spatial variation of the stress components $\sigma_{x x}$ and $\sigma_{x y}$ along the middle line, which marked with the red dashed line in panels (b) and (c). e) The spatial variation of the stress components $\sigma_{\mathrm{nn}}$ and $\sigma_{\mathrm{nt}}$ along one side of the triangle marked with the black dashed line in panels (b) and (c), where $n$ and $t$ represent the normal and tangential direction, respectively. f) The distribution for the trace of stress tensor according to the inclusion theory of Eshelby.

strain field, which can help experimentally clarify the fundamental relationship between physical properties and the mechanical strain. For applications, via internal stress assisted strain engineering the specific nonuniform stress $\backslash$ strain states could be obtained for realizing some interesting theoretical predictions such as the pseduomagnetic field and funnel effect of excitons. Even more amazing, as there is no characteristic size in Elsheby inclusion theory, the stress and strain distribution only depends on the shape of the inclusion, which suggests that the stress or strain gradient could be controlled precisely through changing the size of the inclusion region. As a result, the magnitude of pseudomagneitc field could be accurately designed. Internal stress assisted strain engineering opens an avenue to the practical applications for strain engineering in 2D materials, which injects new vitalities into this field, and with the rapid progress in experiments, the bright prospect of strain engineering should be worth looking forward to.

\section{Experimental Section}

MD Simulations Details: MD simulations were performed using LAMMPS ${ }^{[56]}$ based on the adaptive intermolecular reactive empirical bond-order (AIREBO) potential, ${ }^{[57]}$ with the interaction cutoff parameter set to $2 \AA .{ }^{[58,59]}$ All graphene, graphane, and in-plane grahene/graphane heterostructure samples (about $50 \mathrm{~nm} \times 50 \mathrm{~nm}$ ) used in our simulations were prepared by running NPT dynamics (where the number of particles $N$, pressure $P$, and temperature $T$ of the system are specified) at $1 \mathrm{~K}$ for 100 ps to allow the relaxation of the simulation box dimensions to attain the zero stress state, and then the biaxial or uniaxial tension was applied with a strain rate of $0.001 \mathrm{ps}^{-1}$. Note that a time step of $1 \mathrm{fs}$, periodic boundary condition, and a temperature of $1 \mathrm{~K}$ are used in all of our simulations.

Besides, the atomic structures near the interface obtained by MD simulations and first principle calculations are carefully compared (details could be found in the Supporting Information), which suggests that MD simulations could well predict the graphene/graphane heterostructures even near the interface.

First Principle Calculations Details: The geometrical configurations and electric band structures of monolayer $\mathrm{MoS}_{2}$ are obtained selfconsistently by using the projector augmented wave pseudopotential method implemented in the VASP package, ${ }^{[60]}$ and the exchangecorrelation potential is treated with the hybrid functional HSE06. ${ }^{[47,48]}$ In these calculations, energy cutoff, k-mesh, and convergence criteria are set to be $350 \mathrm{eV}, 12 \times 21 \times 1$, and $10^{-5} \mathrm{eV}$, respectively, and the maximum allowed force on each atom for structure optimization is less than $10^{-3} \mathrm{eV} \AA^{-1}$.

Quantify Mechanical Quantities used in Eshebly Inclusion Theory: With graphene and graphane sharing the same carbon frame at the beginning, the eigenstrain, which describes the variation of the inclusion's volume when constraints from the matrix are relieved, could be estimated by comparing the sizes of the simulation boxes for these two systems after sufficient relaxations in MD simulations. As both the length and width of the simulation box of graphene are $-4.47 \%$ smaller than that of graphane, the eigenstrain is set to $-4.47 \%$ for graphene when including in graphane. After that, tensile MD simulations for graphene and graphane are preformed. The corresponding stress-strain curves are shown in Figure 1b, and also Young's modulus and Poisson's ratio could be obtained. To be specific, they are $960 \mathrm{GPa}$ and 0.16 for graphene, $71.5 \mathrm{GPa}$ and 0.07 for graphane, and are consistent with previous studies. ${ }^{[61,62]}$ Note that the Poisson's ratio for graphane can not 
be accurately captured in MD simulations owing to the slight rippling originating from lack of the mirror symmetry with respect to the middle plane, and its value is taken from the pervious first principles result. [62]

Numerical Solution of Inclusion Problem: The inclusion problem depicts an infinite elastic matrix containing an inclusion with a uniform stress or strain field $\sigma_{i j}^{0}=L_{i j k l} \varepsilon_{k l}^{0}$ at infinity, where $L_{i j k l}$ are the elastic constants of the matrix. According to continuum mechanics, it could be concluded as equilibrium equations (Note that in the following derivations, a suffix preceded by a comma denote differentiation. For example, $\sigma_{i j, j}=\partial \sigma_{i j} / \partial x_{j}$ and $\left.\varepsilon_{i j, j}=\partial \varepsilon_{i j} / \partial x_{j}\right)$

$\sigma_{i j, j}=0$

the stress or strain boundary conditions at infinity

$\sigma_{i j, j}=\sigma_{i j, j}^{0}$ or $\varepsilon_{i j, j}=\varepsilon_{i j, j}^{0}$

the connecting conditions at the interface between the inclusion $(+)$ and matrix $(-)$

$\left\{u_{i}^{+}=u_{i}^{-}\right.$,

$\left\{\sigma_{i j}^{+} n_{j}=\sigma_{i j}^{-} n_{j}\right.$

and the constitutive equations

$\int \sigma_{i j}=L_{i j k l} \varepsilon_{k l}$, (in the matirx)

$\left\{\sigma_{i j}=L_{i j k l}^{c} \varepsilon_{k l}\right.$, (in the inclusion)

where $L_{i j k l}^{c}$ are the elastic constants of the inclusion.

In this article, the inclusion problem is solved numerically based on the complex variable method, and with conformal transformation arbitrary boundary shapes could be handled. Besides, in this method either the strain or stress boundary condition at infinity could be set as required.

Calculation of Pseudomagnetic Field: A 2D strain field could induce a gauge field ${ }^{[10]}$

$A=\frac{\beta}{a}\left(\begin{array}{c}\varepsilon_{x x}-\varepsilon_{y y} \\ -2 \varepsilon_{x y}\end{array}\right)$

where $\beta \approx 2$ and $a$ represent the lattice constant. Note that the $x$-axis should be parallel to the zigzag direction. The corresponding magnetic field, called pseudomagnetic field, could be given as

$B_{S}=\frac{\partial A_{y}}{\partial x}-\frac{\partial A_{x}}{\partial y}$

With the stress field in MD simulations, the strain field could be obtained in terms of constitutive relation, then by taking advantaging of Equations (6) and (7), the distribution of pseudomagnetic field could be calculated.

\section{Supporting Information}

Supporting Information is available from the Wiley Online Library or from the author.

\section{Acknowledgements}

The research was supported by the National Natural Science Foundation of China (Grant Nos. 11602272, 11602270, 11021262, 11172303,
11132011, and 11532013), the National Basic Research Program of China ("973" Project) (Grant No. 2012CB937500), and the Strategic Priority Research Program of the Chinese Academy of Sciences (Grant No. XDB22040503).

\section{Conflict of Interest}

The authors declare no conflict of interest.

\section{Keywords}

2D materials, Eshelby inclusion theory, in-plane heterostructures, MD simulations, strain engineering

Received: October 9, 2017

Revised: January 22, 2018

Published online:

[1] K. S. Novoselov, A. K. Geim, S. V. Morozov, D. Jiang, Y. Zhang, S. V. Dubonos, I. V. Grigorieva, A. A. Firsov, Science 2004, 306, 666.

[2] Q. H. Wang, K. Kalantar-Zadeh, A. Kis, J. N. Coleman, M. S. Strano, Nat. Nanotechnol. 2012, 7, 699.

[3] M. Xu, T. Liang, M. Shi, H. Chen, Chem. Rev. 2013, 113, 3766.

[4] Z. Sun, H. Chang, ACS Nano 2014, 8, 4133.

[5] K. Liu, J. Wu, J. Biomed. Mater. Res. 2015, 31, 832.

[6] J. R. Sánchez-Pérez, C. Boztug, F. Chen, F. F. Sudradjat, D. M. Paskiewicz, R. Jacobson, M. G. Lagally, R. Paiella, Proc. Natl. Acad. Sci. USA 2011, 108, 18893.

[7] P. Johari, V. B. Shenoy, ACS Nano 2012, 6, 5449.

[8] V. M. Pereira, A. C. Neto, Phys. Rev. Lett. 2009, 103, 046801.

[9] S.-M. Choi, S.-H. Jhi, Y.-W. Son, Phys. Rev. B 2010, 81, 081407.

[10] F. Guinea, M. Katsnelson, A. Geim, Nat. Phys. 2010, 6, 30.

[11] W. S. Yun, S. Han, S. C. Hong, I. G. Kim, J. Lee, Phys. Rev. B 2012, 85, 033305

[12] R. Roldán, A. Castellanos-Gomez, E. Cappelluti, F. Guinea, J. Phys.: Condens. Matter 2015, 27, 313201.

[13] J. Feng, X. Qian, C.-W. Huang, J. Li, Nat. Photonics 2012, 6, 866.

[14] P. San-Jose, V. Parente, F. Guinea, R. Roldán, E. Prada, Phys. Rev. X 2016, 6, 031046.

[15] Z. Liu, L. Ma, G. Shi, W. Zhou, Y. Gong, S. Lei, X. Yang, J. Zhang, J. Yu, K. P. Hackenberg, A. Babakhani, J. C. Idrobo, R. Vajtai, J. Lou, P. M. Ajayan, Nat. Nanotechnol. 2013, 8, 119.

[16] T. Gao, X. Song, H. Du, Y. Nie, Y. Chen, Q. Ji, J. Sun, Y. Yang, Y. Zhang, Z. Liu, Nat. Commun. 2015, 6, 6835.

[17] Q. Wu, S. K. Jang, S. Park, S. J. Jung, H. Suh, Y. H. Lee, S. Lee, Y. J. Song, Nanoscale 2015, 7, 7574.

[18] J. H. Meng, X. W. Zhang, H. L. Wang, X. B. Ren, C. H. Jin, Z. G. Yin, X. Liu, H. Liu, Nanoscale 2015, 7, 16046.

[19] M. Liu, Y. Li, P. Chen, J. Sun, D. Ma, Q. Li, T. Gao, Y. Gao, Z. Cheng, X. Qiu, Nano Lett. 2014, 14, 6342.

[20] S. M. Kim, A. Hsu, P. Araujo, Y.-H. Lee, T. Palacios, M. Dresselhaus, J.-C. Idrobo, K. K. Kim, J. Kong, Nano Lett. 2013, 13, 933.

[21] G. H. Han, J. A. Rodríguez-Manzo, C.-W. Lee, N. J. Kybert, M. B. Lerner, Z. J. Qi, E. N. Dattoli, A. M. Rappe, M. Drndic, A. C. Johnson, ACS Nano 2013, 7, 10129.

[22] L. Liu, J. Park, D. A. Siegel, K. F. McCarty, K. W. Clark, W. Deng, L. Basile, J. C. Idrobo, A.-P. Li, G. Gu, Science 2014, 343, 163.

[23] X. Duan, C. Wang, J. C. Shaw, R. Cheng, Y. Chen, H. Li, X. Wu, Y. Tang, Q. Zhang, A. Pan, Nat. Nanotechnol. 2014, 9, 1024.

[24] Y. Gong, J. Lin, X. Wang, G. Shi, S. Lei, Z. Lin, X. Zou, G. Ye, R. Vajtai, B. I. Yakobson, Nat. Mater. 2014, 13, 1135. 
[25] C. Huang, S. Wu, A. M. Sanchez, J. J. Peters, R. Beanland, J. S. Ross, P. Rivera, W. Yao, D. H. Cobden, X. Xu, Nat. Mater. 2014, 13, 1096.

[26] M.-Y. Li, Y. Shi, C.-C. Cheng, L.-S. Lu, Y.-C. Lin, H.-L. Tang, M.-L. Tsai, C.-W. Chu, K.-H. Wei, J.-H. He, Science 2015, 349, 524.

[27] J. D. Eshelby, Proc. R. Soc. London, Ser. A 1957, 241, 376.

[28] P. Sessi, J. R. Guest, M. Bode, N. P. Guisinger, Nano Lett. 2009, 9 , 4343.

[29] J. O. Sofo, A. S. Chaudhari, G. D. Barber, Phys. Rev. B 2007, 75, 153401.

[30] M. T. Lusk, L. D. Carr, Phys. Rev. Lett. 2008, 100, 175503.

[31] J. Lahiri, Y. Lin, P. Bozkurt, I. I. Oleynik, M. Batzill, Nat. Nanotechnol. 2010, 5, 326.

[32] J. Eroms, D. Weiss, New J. Phys. 2009, 11, 095021.

[33] J. Bai, X. Zhong, S. Jiang, Y. Huang, X. Duan, Nat. Nanotechnol. 2010, 5, 190.

[34] M. Kim, N. S. Safron, E. Han, M. S. Arnold, P. Gopalan, Nano Lett. 2010, 10, 1125

[35] Y.-W. Son, M. L. Cohen, S. G. Louie, Phys. Rev. Lett. 2006, 97, 216803.

[36] R. Balog, B. Jørgensen, L. Nilsson, M. Andersen, E. Rienks, M. Bianchi, M. Fanetti, E. Lægsgaard, A. Baraldi, S. Lizzit, Nat. Mater. 2010, 9, 315

[37] L. Ci, L. Song, C. Jin, D. Jariwala, D. Wu, Y. Li, A. Srivastava, Z. Wang, K. Storr, L. Balicas, Nat. Mater. 2010, 9, 430.

[38] N. Levy, S. Burke, K. Meaker, M. Panlasigui, A. Zettl, F. Guinea, A. C. Neto, M. Crommie, Science 2010, 329, 544.

[39] A. Castellanos-Gomez, R. Roldan, E. Cappelluti, M. Buscema, F. Guinea, H. S. van der Zant, G. A. Steele, Nano Lett. 2013, 13, 5361.

[40] H. Li, A. W. Contryman, X. Qian, S. M. Ardakani, Y. Gong, X. Wang, J. M. Weisse, C. H. Lee, J. Zhao, P. M. Ajayan, J. Li, H. C. Manoharan, X. Zheng, Nat. Commun. 2015, 6, 7381.

[41] Y. Y. Hui, X. Liu, W. Jie, N. Y. Chan, J. Hao, Y.-T. Hsu, L.-J. Li, W. Guo, S. P. Lau, ACS Nano 2013, 7, 7126

[42] L. Yang, X. Cui, J. Zhang, K. Wang, M. Shen, S. Zeng, S. A. Dayeh, L. Feng, B. Xiang, Sci. Rep. 2014, 4, 5649.
[43] H. J. Conley, B. Wang, J. I. Ziegler, R. F. Haglund Jr., S. T. Pantelides, K. I. Bolotin, Nano Lett. 2013, 13, 3626.

[44] C. Zhu, G. Wang, B. Liu, X. Marie, X. Qiao, X. Zhang, X. Wu, H. Fan, P. Tan, T. Amand, Phys. Rev. B 2013, 88, 121301.

[45] Q. Ji, Y. Zhang, T. Gao, Y. Zhang, D. Ma, M. Liu, Y. Chen, X. Qiao, P.-H. Tan, M. Kan, Nano Lett. 2013, 13, 3870.

[46] J. P. Perdew, K. Burke, M. Ernzerhof, Phys. Rev. Lett. 1996, 77, 3865.

[47] J. Heyd, G. E. Scuseria, M. Ernzerhof, J. Chem. Phys. 2003, 118, 8207.

[48] J. Heyd, G. E. Scuseria, M. Ernzerhof, J. Chem. Phys. 2006, 124, 219906.

[49] E. P. Nguyen, B. J. Carey, J. Z. Ou, J. van Embden, E. D. Gaspera, A. F. Chrimes, M. J. Spencer, S. Zhuiykov, K. Kalantar-zadeh, T. Daeneke, Adv. Mater. 2015, 27, 6225.

[50] J. D. Wood, S. A. Wells, D. Jariwala, K.-S. Chen, E. Cho, V. K. Sangwan, X. Liu, L. J. Lauhon, T. J. Marks, M. C. Hersam, Nano Lett. 2014, 14, 6964

[51] C. Huang, S. Wu, A. M. Sanchez, J. J. Peters, R. Beanland, J. S. Ross, P. Rivera, W. Yao, D. H. Cobden, X. Xu, Nat. Mater. 2014, 13, 1096.

[52] Y. Shi, W. Zhou, A. Y. Lu, W. Fang, Y. H. Lee, A. L. Hsu, S. M. Kim, K. K. Kim, H. Y. Yang, L. J. Li, J. C. Idrobo, J. Kong, Nano Lett. 2012, 12,2784

[53] S. Bertolazzi, J. Brivio, A. Kis, ACS Nano 2011, 5, 9703.

[54] K. Liu, Q. Yan, M. Chen, W. Fan, Y. Sun, J. Suh, D. Fu, S. Lee, J. Zhou, S. Tongay, Nano Lett. 2014, 14, 5097.

[55] F. Zeng, W.-B. Zhang, B.-Y. Tang, Chin. Phys. B 2015, 24, 097103.

[56] S. Plimpton, J. Comput. Phys. 1995, 117, 1.

[57] S. J. Stuart, A. B. Tutein, J. A. Harrison, J. Chem. Phys. 2000, 112, 6472.

[58] O. Shenderova, D. Brenner, A. Omeltchenko, X. Su, L. Yang, Phys. Rev. B 2000, 61, 3877.

[59] Z. Xu, J. Comput. Theor. Nanosci. 2009, 6, 625

[60] G. Kresse, J. Furthmuller, Phys. Rev. B 1996, 54, 11169.

[61] Q. X. Pei, Y. W. Zhang, V. B. Shenoy, Carbon 2010, 48, 898.

[62] M. Topsakal, S. Cahangirov, S. Ciraci, Appl. Phys. Lett. 2010, 96 , 091912. 